\title{
Gestión de Stakeholders para la Productividad y Competitividad por Gobiernos Locales
}

\section{Stakeholders Management. Application in Local Governments for Productivity and Competitiveness}

\section{AUTOR 1:}

Rojas Palacios Luis Eden:

Doctorado en Gestión Pública y Gobernabilidad, Universidad César Vallejo, Perú. ORCID: https://orcid.org/00000002-9285-8367 Correo: Irojaspa12@ucvvirtual.ed u.pe
Recibido: 26 de junio 2021

Aprobado: 28 de septiembre 2021

\section{RESUMEN:}

En el Perú existen escasos trabajos de investigación sobre gestión de stakeholders en los gobiernos locales, es por ello, se hizo una búsqueda sistemática de literatura en base a datos conocidas, usando palabras claves y análisis de la información. El objetivo fue identificar las tendencias en la Gestión de Stakeholders o grupo de interés implementados en contextos de gestión pública para aumentar la productividad social y competitividad. La revisión sistemática realizada llevó a identificar a 21 artículos publicados; los mismos que fueron sometidos a un proceso de lectura y análisis. Se accedió a diversas bases de datos (Scopus, Scielo, Google Académico, etc.) que contenían revistas indizadas como: ICONO 14, La investigación de: Información, Archivística, bibliotecología, Signo y Pensamiento, Gestionamiento y Política Pública, Innovar, Perspectivas, Gestión y Pensamiento y la Revista Venezolana de Gerencia, donde se obtuvo información utilizando operadores booleanos. Por lo tanto, los resultados obtenidos muestran, que existe una tendencia al diálogo y el compromiso que asume cada stakeholders y ello aumentará la productividad y a su vez la competitividad en la mejora de niveles de desarrollo, generando impactos positivos y sostenibles en los gobiernos locales.

Palabras clave: Gestión de stakeholders, gobierno local, productividad, competitividad.

\begin{abstract}
:
In Peru there are few research works on stakeholder management in local governments, therefore, a systematic literature search was carried out based on known data, using keywords and information analysis. The objective was to identify trends in stakeholder management implemented in public management contexts to increase social productivity and competitiveness. The systematic review carried out led to the identification of 21 published articles; the same ones that were subjected to a reading and analysis process. Various databases were accessed (Scopus, Scielo, Google Scholar, etc.) that contained indexed journals such as: ICON 14, Library Research: Archivonomy, Library Science and Information, Sign and Thought, Management and Public Policy, Innovate, Perspectives, Gestión y Pensamiento and Revista Venezolana de Gerencia, where information was obtained using Boolean operators. Therefore, the results obtained show that there is a tendency to dialogue and commitment assumed by each stakeholder and this will increase productivity and in turn competitiveness in improving development levels, generating positive and sustainable impacts on local governments.
\end{abstract}

Keywords: Stakeholder management, local government, productivity, competitiveness. 


\section{INTRODUCCIÓN}

El objetivo fue identificar las tendencias en la Gestión de Stakeholders o grupo de interés implementados en contextos del ámbito público con relación al incremento en la productividad y la competitividad de los gobiernos locales; cabe precisar que no existen acuerdos sobre la definición de lo que es competitividad y aunque es vista desde diversas perspectivas, es claro que de alguna manera relaciona la capacidad de la organización para enfrentar a sus contrapartes con éxito, cuando brindar sus servicios (Rubio y Baez, 2008).

Partimos de Stakeholders Theory o Teoría de los grupos de interesados, que ha adquirido particular relevancia en los últimos años. Desde que Edward R. Freeman (1984: 25) publicó su estudio "Strategic management: A stakeholder approach", en el que señaló que los "partícipes son cualquier grupo o individuo que influye o es afectado por la actividad o los objetivos de la compañía, los que pueden adoptar un sin fin de formas y clasificaciones y que, estan determinados por las propias características y dimensiones de la empresa".

Cabe como base a la acepción de Stakeholders, llamarlos Grupos de interés como exigencia ética y de responsabilidad (Fernández \& Bajo, 2012). Que implica un proceso de planificación colaborativa que involucra a todas las partes interesadas y afectadas, así mismo, el intercambio continuo de información y el diálogo con los grupos de partes interesadas están proporcionando creación de capacidad (Rutherford et al., 2005).

Involucrar a las partes interesadas en el desarrollo y mejora de la gestión repercute en los índices de calidad de vital de los ciudadanos del territorio local; sin embargo, la toma de decisiones, no siempre acertadas de las autoridades, conlleva a una gestión de limitados resultados. La importancia de autoridades involucradas que toman en cuenta las competencias de los stakeholders, brinda oportunidades a su gestión. Además, se fortalece en los capacitados, el compromiso; ello consiente el aumento de productividad y mejora en la calidad de gestión.

En este marco, se reconoce que las instituciones que logran desarrollar la competitividad promueven la colaboración, coordinación (Zurita \& Nuflo, 2014) y el aumento significativo de la competencia, a favor de la productividad en las instituciones públicas (Ghulam \& Mousa, 2019), así mismo, los gobiernos locales afectan el desempeño de las partes interesadas (Deng et al., 2019), mayor cualificación en la población, mayor será la productividad, cuanto mayor sea el grado de inclusión social, mayor será la participación de los stakeholders (Alsudairi \& Kamal, 2009). 
Frente a este desafío, cabe preguntarse ¿Cuáles son las tendencias en la Gestión de Stakeholders o grupo de interés implementados en contextos de gestión pública para aumentar la productividad social y competitividad?

La presente investigación de revisión de la literatura referida a la gestión de stakeholders para aumentar la productividad y competitividad aplicada desde lo que le corresponde a los gobiernos locales; en una perspectiva de la nueva gestión pública que incorpora renovadas formas de trabajo, lo que permite el derecho de ser escuchados, asumiendo compromisos, aumentando su bagaje cultural, valores éticos, reputación, logrando de esta manera que el gobierno local desarrolle sus funciones de manera efectiva para lograr un mejor desempeño en el sector público productivo y competitivo. El objetivo fue identificar las tendencias en la Gestión de Stakeholders o grupo de interés implementados en contextos de gestión pública.

Es sabido que, la gestión de stakeholders en las organizaciones ha encontrado en dichos entornos nuevas e innovadoras oportunidades de negocios debido a herramientas que han permitido fomentar conversaciones con nuevos actores, logrando hacerlos partícipes en las estrategias comunicativas propias, convirtiédose en una buena práctica. Como consecuencia se considera que se han convertido en stakeholders auténticos" (Moya \& Moya, 2020); otro aspecto relevante son las razones y motivaciones que llevan a una divulgación de la informativa relacionada a Responsabilidad Social (RS) por parte de los municipios (Nevado Gil et al., 2019).

En esta línea, algunas experiencias respecto a la heterogeneidad de los stakeholders, como es el caso de TV3, dieron cuenta de la importancia que tienen las difersas formas de comunicar los logros alcanzados (Toro \& Ferré-Pavia, 2017). Visibilizando, que la RSE adquiere una foma complejo de la comprensión de la labor de dirección, y actuar coherente de la búsqueda de sostenibilidad.

Po otro lado, los gobiernos locales, más allá, del tema económico enfrentan dos aspectos que se plantean de manera reiterada respecto a su naturaleza; y es, el escaso conocimiento respecto a su esencia y lo que significan; y la convicción de que las características de las personas que se encuentran al frente de las iniciativas y organizaciones que se ubican en el territorio determinan muchas de las particularidades de los servicios y acciones que allí se llevan a cabo" (Szafran, 2016).

El mencionado desafío debe de constar con el estricto compromiso por parte del Estado descentralizado, para no obstaculizar el buen desarrollo del movilismo endógeno, incorporando así una política localizada que pueda utilizar distintos y nuevos instrumentos implicando la adaptación a la adopción de los criterios epistemológicos locales (López \& Bustamante, 2016). 
Además, otro elemento importante es la productividad de stakeholders, que cuenta con basto estudio y estadística, que ha logrado medir la competividad, permitiendo la identificación de factores inhibitorios del nivel de productividad en las economías locales, para minimizalos e impulsar el desempeño en el área económico, a favor de la planeación económica, y facilitación de trabajos de coordinación eficaz con las áreas de atención; logrando una aplicación de estrategias, acciones que permitan elevar y mejorar los índices de competividad territorial y realizar una planeación económica estable (Huber Bernal \& Mungaray Lagarda, 2017).

Visto esto, desde la perspectiva del que hacer administrativo cotidiano, la caracterización de procesos ambientes y laborales que se pueden percibir en un entorno diferencial como Guayaquil, muestran la incidencia que tienen en la productividad de los colaboradores, a su vez estos son tomados como una referencia para poder comprender la conducta de la organización para lograr establecer las estrategias gestacionales enfocadas en el manejo del talento u recurso humano y mejorar los ya conocidos índices de productividad de los colaboradores, y la empresa en general pudiendo conseguir así el correcto nivel de competencia (Ganga \& Villacís, 2018).

Por otro lado, este estudio tiene diversas implicaciones para las organizaciones, puesto que la aplicación de un sistema basado en la identificación de competencias y su posterior desarrollo en los trabajadores mediante planes formativos puede colocar a la organización en una posición más competitiva al aumentar la productividad, a la vez que los trabajadores crecen personal y profesionalmente" (Hinojo Lucena., Aznar Díaz., Romero Rodríguez, 2020). Sólo los sectores que son capaces de aumentar su productividad a través de la cooperación técnica pueden beneficiarse de la transferencia de tecnología (Bruhn et al., 2017).

La competitividad de stakeholders se manifiesta a través del análisis competencial interno, en el cual se encuentran correlaciones significativas entre la calidad y la mejora de servicios brindados; ello son trámites sencillos y rápidos los que mejoran la atención del público y proporcionan la información al cliente a disponibilidad de los manuales dados por servidores públicos. (Gómez Díaz, 2016); a su vez, en medida de que los índices nacionales indiquen que la competividad y describan de modo claro, exhaustivo y transparente desde los anexos de metodología, los cambios producidos en la medición de competividad territorial, será de una utilidad mayor como el seguimiento y el apoyo para con las políticas públicas (Huber Bernal \& Mungaray Lagarda, 2017).

Las organizaciones lo que buscan es una mejoría y tras obtenerla se logra un mejorar en la calidad de vital del equipo colaborador, a consecuencia en dicha localidad, permitiendo así la innovación de distintas maneras de acorde a la estructura 
empresarial, en determinadas empresas en las que se ponen en marcha los cambios para una mejor atención del cliente, el cual ha sido un factor de suma importancia (Luz et al., 2019); aunque el avance académico y práctico de conceptos de conectividad organizacional, en el diálogo de apropiación realizada en el estudio de los procesos de políticas públicas que lo hallamos siempre en constante cambio y dinámica permanente (Ruano lbarra et al., 2019); la responsabilidad pública en la planificación va acompañado de un renovado interés en generar apoyo público para la implementación de la planificación estratégica (Moutinho \& Rabechini Junior, 2020).

\section{MÉTODO}

La metodología empleada fue propia de la "Revisión Sistemática" en bases de datos como Scopus, a fin de identificar, evaluar y lograr interpretar la investigación relevante a disposición a partir de la pregunta de investigación planteada ¿Cuál es la tendencia sobre la Gestión de Stakeholders para la Productividad y Competitividad de los Gobiernos Locales? Se adoptó para este estudio algunos procesos propuestos por Kitchenham et al. (2004). El resultado del análisis de las principales fuentes, son estudios revisados por pares documentados y respaldados por criterios de revisión de las revistas académicas. Se aplicó un protocolo de búsqueda en la base de datos de Scopus, Redalyc, Scielo procesos:

Diseño de la estrategia de búsqueda: Primer protocolo de búsqueda utilizado en la base datos Scopus :( título-abs-key (partes interesadas y gestión) título-abs-key (productividad) y título-abs-key (competencia $\mathrm{Y}$ por $\mathrm{Y}$ local de $\mathrm{Y}$ gobiernos). Segundo base de datos Scopus Protocolo de búsqueda: (title-ab-key (social and policy) and title-ab-key (productivity) and title-ab-key (competition) and title-ab-key (local AND governments ) ).

Ejecución de la búsqueda: La metodología empleada fue la revisión bibliomética de publicaciones en bases de datos (Scopus, Redalyc, Scielo, etc.) utilizando palabras claves tanto en inglés como en español; además se utilizó operadores booleanos como "AND"y "OR".

La validez de este tipo de recursos radica en que se obtienen a través de fuentes confiables. La pregunta de investigación establecida para el marco metodológico fue la siguiente: ¿Cuál es la tendencia sobre la Gestión de Stakeholders para la Productividad y Competitividad de los Gobiernos Locales? 


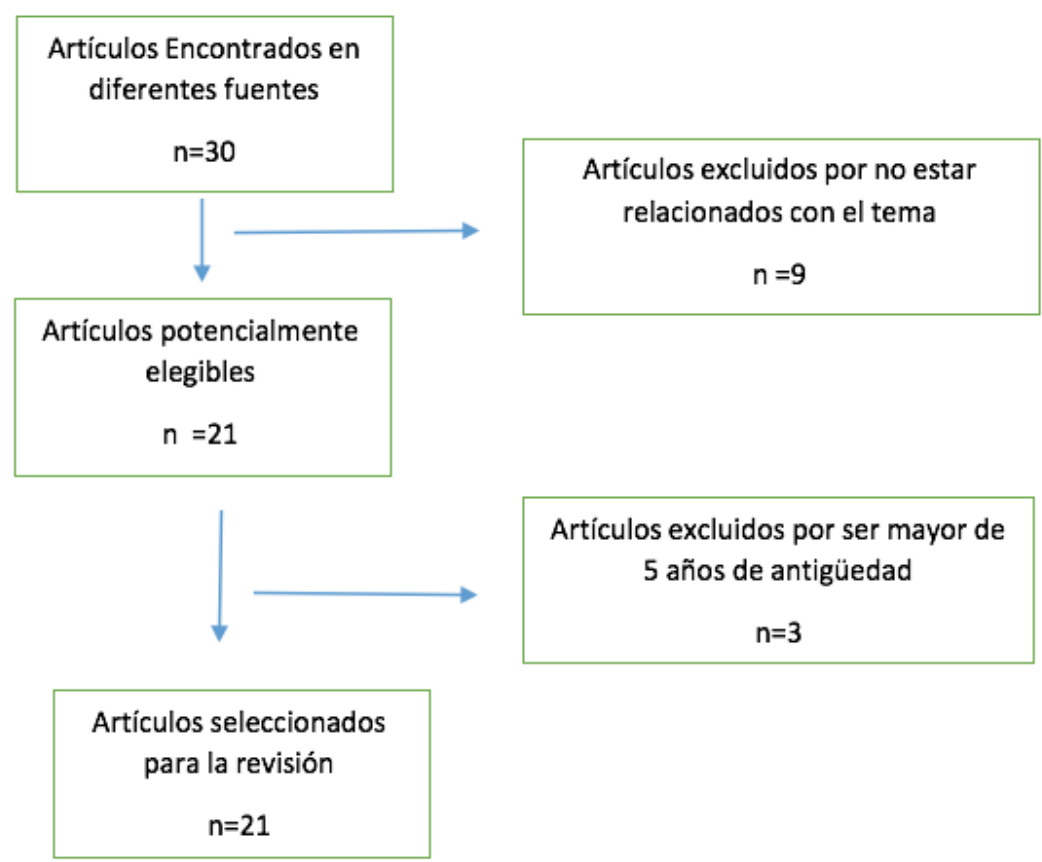

Figura 1. Representación gráfica de búsqueda de información y selección sobre artículos.

Este permitió identificar a través del protocolo de búsqueda: 8 publicaciones en Scopus, 10 publicaciones en Scielo y 12 publicaciones en Redalyc entre los años 2016 al 2020.

Análisis de los resultados permitió identificar 21 artículos relacionados con el campo temático en diferentes revistas que abordaban el campo temático de Responsabilidad Social y Stakeholder o grupos de interesados, los cuales fueron exportados al gestor de información Mendeley.

\section{Tabla 1.}

Palabras claves para la búsqueda de artículos científicos.

\begin{tabular}{|c|c|c|}
\hline Palabra Clave & Español & Inglés \\
\hline Gestión de stakeholders & $\begin{array}{c}\text { Gestión de } \\
\text { stakeholders }\end{array}$ & Stakeholder management \\
\hline Gobierno local & Gobierno local & local government \\
\hline $\begin{array}{l}\text { Productividad } \\
\text { Competitividad }\end{array}$ & $\begin{array}{l}\text { Productividad social } \\
\text { Competitividad }\end{array}$ & $\begin{array}{c}\text { productivity } \\
\text { competitiveness }\end{array}$ \\
\hline
\end{tabular}

La información consignada en la tabla 1, presenta las palabras claves y la traducción al idioma con que se hizo la búsqueda, en las bases de datos. Scopus, Google académico y Redalyc. Siendo estas en español, Gestión, Gobierno local, Productividad social 
Competitividad y en inglés tales como, Stakeholder management, local government, productivity, competitiveness.

\section{RESULTADOS.}

En los gobiernos locales la implementación de gestión de stakeholders para aumentar la productividad y competitividad es limitado, asimismo se necesita contar con servidores públicos prestos a ser escuchados, asumiendo compromisos, capacitados dispuestos a enfrentar retos y lograr cambios en la gestión municipal.

Tabla 2. Palabras claves para la búsqueda de artículos científicos.

\begin{tabular}{|c|c|c|c|c|c|c|}
\hline $\begin{array}{l}\text { Base } \\
\text { de datos }\end{array}$ & $\begin{array}{l}\text { Título } \\
\text { Artículo }\end{array}$ & del & $\begin{array}{l}\text { Autor } \\
\text { Año }\end{array}$ & País & $\begin{array}{l}\text { Tipo de } \\
\text { estudio }\end{array}$ & Conclusión \\
\hline 1 Scopus & $\begin{array}{l}\text { Hacia un } \\
\text { concepto } \\
\text { stakeholders }\end{array}$ & $\begin{array}{l}\text { nuevo } \\
\text { de }\end{array}$ & $\begin{array}{l}\text { Eugenio Moya } \\
\text { y Julia Moya, } \\
2020 \text {. }\end{array}$ & $\begin{array}{l}\text { Espa } \\
\text { ña }\end{array}$ & $\begin{array}{l}\text { Artículo } \\
\text { Científic } \\
0\end{array}$ & $\begin{array}{l}\text { "Vemos que los } \\
\text { stakeholders nacientes } \\
\text { permiten configurar todos } \\
\text { los mensajes de } \\
\text { contenido que garantizan } \\
\text { la pertinente } \\
\text { transformación } \\
\text { estrategias en las } \\
\text { instituciones" }\end{array}$ \\
\hline
\end{tabular}

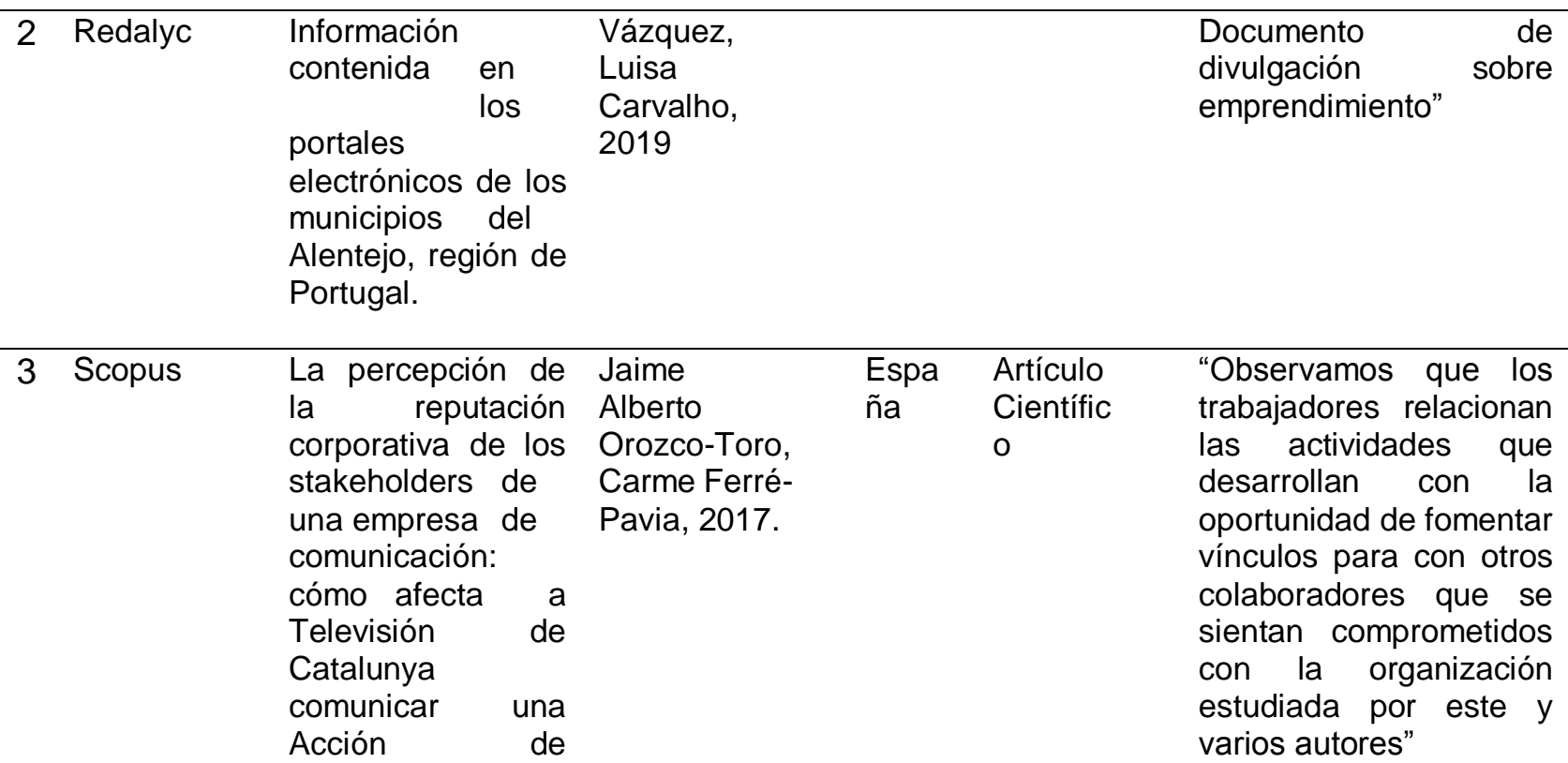




\section{Scopus \\ Información, comunicación y Cultura en la gestión local: la experiencia municipal en un territorio de vulnerabilidad de la ciudad de Montevideo. e de}

Paulina

Szafran, 2016

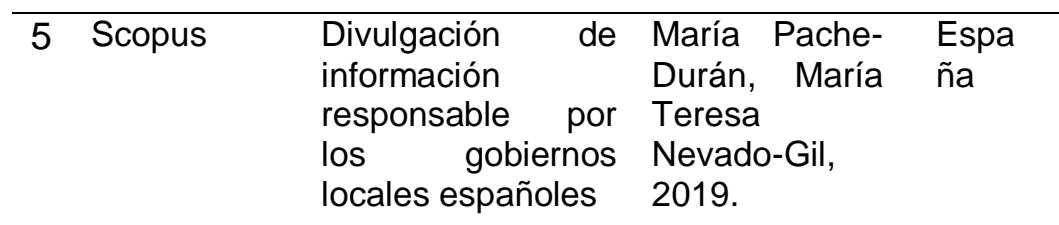

"Vemos la relación entre la información y la comunicación representada en la gestión de cada municipio."

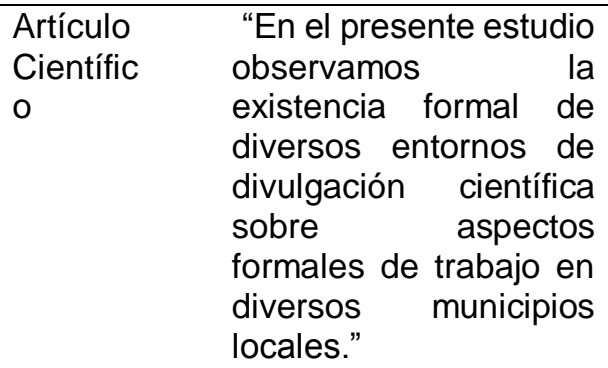
"El presente trabajo
aborda el
emprendurismo
realizado por los
municipios de la región
de Cáceres que
finalmente se llegan a
divulgar mediante
páginas webs"

$\begin{array}{ll}\text { Colo } & \text { Artículo } \\ \text { mbia } & \text { Científic } \\ & 0\end{array}$

"Como un agregado de las prácticas comunicativas unidireccionadas, vemos que la presión diaria a la cual se enfrenta el servidor público para cumplir el marco normativo propio de la organización es determinada como respuesta a los indicadores de gestión"




los trabajadores de Moyano, las grandes y 2018. medianas empresas Guayaquil. pertenencia en los trabajos en equipo."

\begin{tabular}{|c|c|c|c|c|c|}
\hline 1 Scopus & $\begin{array}{l}\text { Factor humano en } \\
\text { la productividad } \\
\text { empresarial: un } \\
\text { enfoque desde el } \\
\text { análisis de las } \\
\text { competencias } \\
\text { transversales. }\end{array}$ & $\begin{array}{l}\text { Francisco } \\
\text { Javier Hinojo } \\
\text { Lucena, } \\
\text { Inmaculada } \\
\text { Aznar Díaz, } \\
\text { José María } \\
\text { Romero } \\
\text { Rodríguez, } \\
2020 .\end{array}$ & $\begin{array}{l}\text { Espa } \\
\text { ña }\end{array}$ & $\begin{array}{l}\text { Artículo } \\
\text { Científic } \\
0\end{array}$ & $\begin{array}{l}\text { "La competencia } \\
\text { profesional desarrolla un } \\
\text { papel de gran importancia } \\
\text { en cuanto a la obtención d } \\
\text { objetivos, es por dicha } \\
\text { razón que inciden en la } \\
\text { productividad" }\end{array}$ \\
\hline 1 Redalyc & $\begin{array}{l}\text { Modelo estratégico } \\
\text { de aprendizaje } \\
\text { organizacional } \\
\text { para impulsar la } \\
\text { competitividad } \\
\text { municipal }\end{array}$ & $\begin{array}{l}\text { María del } \\
\text { Rocío Gómez } \\
\text { Díaz, } 2016 .\end{array}$ & $\begin{array}{l}\text { Méxic } \\
\text { o }\end{array}$ & $\begin{array}{l}\text { Artículo } \\
\text { Científic } \\
\text { o }\end{array}$ & $\begin{array}{l}\text { "Podemos referirnos a } \\
\text { dos tipos de } \\
\text { competividad: } \\
\text { Competividad Interna } \\
\text { Competividad externa" }\end{array}$ \\
\hline 1 Scopus & $\begin{array}{l}\text { Innovación } \\
\text { Empresarial: } \\
\text { Factor de } \\
\text { competitividad y } \\
\text { calidad de vida en } \\
\text { Popayán, } \\
\text { Colombia }\end{array}$ & $\begin{array}{l}\text { Saldarriag } \\
\text { a Alazar, } \\
\text { María } \\
\text { Eugenia1 } \\
\text { Guzmán } \\
\text { González, } \\
\text { María } \\
\text { Fernanda2 } \\
\text { Concha } \\
\text { Cerón, } \\
\text { Eduardo } \\
\text { Andrés3, } \\
2019 .\end{array}$ & $\begin{array}{l}\text { Colo } \\
\text { mbia }\end{array}$ & $\begin{array}{l}\text { Artículo } \\
\text { Científic } \\
0\end{array}$ & $\begin{array}{l}\text { "La competencia ha } \\
\text { logrado implementar la } \\
\text { innovación dando como } \\
\text { resultado mejores ideas, } \\
\text { a su vez atendió a } \\
\text { cambios generados por } \\
\text { el entorno." }\end{array}$ \\
\hline 1 Redalyc & $\begin{array}{l}\text { Centro de } \\
\text { competitividad } \\
\text { n Colombia: Análisis } \\
\text { histórico } \\
\text { institucional }\end{array}$ & $\begin{array}{l}\text { Ruano- } \\
\text { lbarra, } \\
\text { Elizabeth1 } \\
\text { Rivera, } \\
\text { Wilfred2 } \\
\text { Alvarado, } \\
\text { Oscar3. } 2019\end{array}$ & $\begin{array}{l}\text { Colo } \\
\text { mbia }\end{array}$ & $\begin{array}{l}\text { Artículo } \\
\text { Científic } \\
0\end{array}$ & $\begin{array}{l}\text { "Como argumento final } \\
\text { se puede afirmar que la } \\
\text { competencia } \\
\text { empresarial } \\
\text { referida a la capacidad } \\
\text { de acopio de conoceres } \\
\text { a través del } \\
\text { enraizamiento de } \\
\text { técnicas innovadoras". }\end{array}$ \\
\hline
\end{tabular}

Es evidente que en Latinoamérica que la tendencia de la experiencia de la práctica de Responsabilidad Social se ha incrementado y países como Colombia, México, España, Ecuador y Uruguay vienen aportando al conocimiento, desde sus buenas prácticas en las que la responsabilidad Social ha trascendido el ámbito privado y empresarial hacia otros, como el público, incorporando en su práctica otras disciplinas, llegando a buscar generar competitividad, para generar productividad en el aspecto social, agregando valor a la articulación con los grupos de interesados o Stakeholdersa tavés de un elemento clave, que es la comunicación. 


\section{CONCLUSIONES.}

Existe una tendencia marcada en latinoamérica respecto al incremento de experiencias en el ámbito público como gobiernos locales, respecto a los stakeholder o grupos de interesados, que ha ido agregando valor a través de la movilización de la productividad y la competitividad, en el ámbito público.

Se identifica que existen aspectos claves como la comunicación entre los stakeholder apoyada por la tecnología digital, variadas experiencias dan cuenta del aumento de la productividad y desempeño, como valor agregado para las políticas públicas el ámbito de la gestión gubernamental, con gran nivel de movilizar impacto en el territorio.

Se identifica que existen aspectos claves como la comunicación entre los stakeholder apoyada por la tecnología digital para fortalecer la comunicación y experiencias que dan cuenta del aumento de la productividad y desempeño, como valor agregado para las políticas públicas el ámbito de la gestión pública, con gran nivel movilizar de impacto en el territorio.

A manera de reflexión, los gobiernos locales, aún enfrentan desafíos que deben minimizar con acciones a largo plazo con mirada de territorio; siendo un reto, desde perspectivas teóricas adaptadas a la gestión pública, tanto en el ámbito, social, económico y sustentabilidad, logrando que la gestión de los stakeholders desarrolla sus funciones de manera efectiva.

\section{REFERENCIAS BIBLIOGRÁFICAS}

Alsudairi, M., \& Kamal, M. (2009). "ICT policy" for ICT service management - Role of business organisations in National Reforms Agenda for services sector. https://cutt.ly/1YIXE6S

Bruhn, N. C. P., Calegário, C. L. L., Carvalho, F. D. M., Campos, R. S., \& Santos, A. C. D. (2017). Mergers and acquisitions in Brazilian industry: a study of spillover effects. International Journal of Productivity and Performance Management, 66(1), 51-77. https://doi.org/10.1108/IJPPM-11-2014-0179

Cagica Carvalho, L., Pérez Calderón, E., \& Pache Durán, M. (2019). Implicación de los gobiernos locales en la promoción del emprendimiento: evidencia para España. Revista Española de Documentación Científica, 42(1), 226. https://doi.org/10.3989/redc.2019.1.1559 
Deng, J., Zhang, N., Ahmad, F., \& Draz, M. U. (2019). Local government competition, environmental regulation intensity and regional innovation performance: An empirical investigation of Chinese provinces. International Journal of Environmental Research and Public Health, 16(12). https://doi.org/10.3390/ijerph16122130

Fernández, J. L., \& Bajo, A. (2012). La Teoría del Stakeholder o de los Grupos de Interés, pieza clave de la RSE, del éxito empresarial y de la sostenibilidad. Adresearch Esic International Journal of Communication Research, 6(6), 130-143. https://doi.org/10.7263/adr.rsc.006.07

Ganga, F., \& Villacís, H. (2018). Factores individuales y grupales que influyen en la productividad de los trabajadores de las grandes y medianas empresas de Guayaquil. Revista Perspectivas, 42, 97-122.

Ghulam, Y., \& Mousa, W. I. (2019). Estimation of productivity growth in the Saudi higher education sector. Technological Forecasting and Social Change, 149. https://doi.org/10.1016/i.techfore.2019.119741

Gómez Díaz, M. (2016). Modelo estrátegico de aprendizaje organizacional para impulsar la competitividad municipal. Revista Científica Pensamiento y Gestión, O(40).

Friedman, M., (1970), "The social responsibility of business is to increase its profit", The New York Times Magazine, september 13th., USA, pp. 32-33, 122-126.

Hinojo Lucena, F. J., Aznar Díaz, I., \& Romero Rodríguez, J. M. (2020). Human factor and business productivity: An approach from the analysis of cross-cutting competences. Innovar, 30(76), 51-62. https://doi.org/10.15446/innovar.v30n76.85194

Huber Bernal, G., \& Mungaray Lagarda, A. (2017). Los índices de competitividad en México. Gestion y Politica Publica, 26(1), 167-218. https://cutt.ly/9YIXBBF

López, A. M., \& Bustamante, E. M. G. (2016). La comunicación y la información en Gobiernos locales: Persistencia de prácticas difusionistas de comunicación en organizaciones gubernamentales del Valle del Cauca (Colombia). Signo $y$ Pensamiento, 35(69), 118-139. https://doi.org/10.11144/Javeriana.syp35-69.cigl

Luz, Z., Salazar, S., Eugenia, M., González, G., Fernanda, M., Cerón, C., \& Andrés, E. (2019). Innovación Empresarial: Factor de competitividad y calidad de vida en Popayán, Colombia. Revista Venezolana de Gerencia, 127-143. https://doi.org/10.37960/revista.v24i2.31486 
Moutinho, J. D. A., \& Rabechini Junior, R. (2020). Project management in the public context: Research field mapping . Revista de Administracao Publica, 54(5), 12601285. https://doi.org/10.1590/0034-761220190327x

Moya, E., \& Moya, J. (2020). Prosum, swarming and transmedia. Towards a new concept of stakeholder. Icono14, 16(2), 25-52. https://doi.org/10.7195/RI14.V16I2.1213

Nevado Gil, M. T., Gallardo-Vázquez, D., \& Carvalho, L. (2019). Emprendimiento en la administración local: un estudio empírico de la información contenida en los portales electrónicos de los municipios del Alentejo, región de Portugal. In Innovar (Vol. 29, Issue 71, pp. 97-112). https://doi.org/10.15446/innovar.v29n71.76398

Pache-Durán, M., \& Nevado-Gil, M. T. (2019). Divulgación de información responsable por los gobiernos locales españoles. Investigación Bibliotecológica: Archivonomía, $\begin{array}{llll}\text { Bibliotecología } \quad e & \text { Información, } & 33(81), & 11 .\end{array}$ https://doi.org/10.22201/iibi.24488321xe.2019.81.58043

Rutherford, R. J., Herbert, G. J., \& Coffen-Smout, S. S. (2005). Integrated ocean management and the collaborative planning process: The Eastern Scotian Shelf Integrated Management (ESSIM) Initiative. Marine Policy, 29(1), 75-83. https://doi.org/10.1016/j.marpol.2004.02.004

Szafran, P. (2016). Información, comunicación y cultura en la gestión local: la experiencia municipal en un territorio de vulnerabilidad de la ciudad de Montevideo. Investigacion Bibliotecologica, $\quad 30(70), \quad 19-39$. https://doi.org/10.1016/i.ibbai.2016.10.002

Toro, J. A. O., \& Ferré-Pavia, C. (2017). The perception of the corporate reputation of the stakeholders from a communication company: How televisió de catalunya is affected by communicating a corporate social responsibility action. In Palabra Clave (Vol. 20, Issue 2). https://doi.org/10.5294/pacla.2017.20.2.8

Zurita, J. C., \& Nuflo, J. P. (2014). The major milestones to constitute SINTONA an institute for collaboration (IFC) (C. J.V., C. N., O. A., S. B., \& W. F. (eds.); pp. 105109). International Institute of Informatics and Systemics, IIIS. https://cutt.ly/xYIChVW 